\title{
PRODUÇÃO DE MASSA SECA, EFICIÊNCIA E RECUPERAÇÃO DO NITROGÊNIO E ENXOFRE PELO CAPIM-TANZÂNIA ADUBADO COM NITROGÊNIO, POTÁSSIO E ENXOFRE ${ }^{1}$
}

\author{
Dry mass production, nitrogen and sulfur efficiency and recovery by tanzania grass fertilized \\ with nitrogen, potassium and sulfur
}

\author{
Kátia Aparecida de Pinho Costa ${ }^{2}$, Aldi Fernandes de Souza França ${ }^{3}$, Itamar Pereira de Oliveira ${ }^{4}$, \\ Francisco Antonio Monteiro ${ }^{5}$, José Alexandre Freitas Barigossi ${ }^{4}$
}

\section{RESUMO}

O experimento foi realizado em um Latossolo Vermelho Escuro, na Fazenda Modelo da Universidade Federal de Goiás, com o objetivo de avaliar o efeito de doses da formulação N: K com o uso do S na produção de massa seca do capim-Tanzânia. Também foram avaliadas a eficiência de conversão em massa seca e a recuperação relativa do $\mathrm{N}$ e do $\mathrm{S}$. A formulação N:K foi aplicada em cobertura, nas doses de 150, 300 e $450 \mathrm{~kg} / \mathrm{ha}$, com adição de enxofre de 20, 40 e $60 \mathrm{~kg} / \mathrm{ha}$. O delineamento experimental foi de blocos completos ao acaso, em esquema fatorial 3 x 3, com três repetições. O período de avaliação da forrageira foi seis meses e as amostras foram coletadas à altura de $30 \mathrm{~cm}$ acima do solo. Melhores ECN e ECS são observadas quando se aplica menores doses de $\mathrm{N}: \mathrm{K}$ e $\mathrm{S}$, mesmo não havendo diferenças significativas nas concentrações destes nutrientes no tecido da planta sob a ação de doses crescentes dos mesmos. Ao contrário, as maiores RRN e RRS foram observadas quando se aplicou $450 \mathrm{~kg} / \mathrm{ha}$ da formulação N:K na presença de $60 \mathrm{~kg} / \mathrm{ha}$ de S.

Termos para indexação: concentração de nutrientes, eficiência da conversão de N e S, recuperação relativa do N e S, Panicum maximum.

\begin{abstract}
The experiment was carried out in a Dark Red Latosol at Fazenda Modelo, Federal University of Goiás, in order to evaluate the effect of $\mathrm{N}: \mathrm{K}$ rates with the use of $\mathrm{S}$ in the production of dry mass of Tanzania grass. Also, the efficiency of $\mathrm{N}$ and $\mathrm{S}$ conversion and recovery in dry mass were evaluated. Rates of 150, 300 and $450 \mathrm{~kg} / \mathrm{ha}$ of N:K aditioning $20,40 \mathrm{and} 60 \mathrm{~kg} / \mathrm{ha}$ of $\mathrm{S}$ were applied as broadcast covering. The experimental design was a randomized complete blocks in a $3 \mathrm{x} 3$ factorial with three replications. The forage evaluation period was six months and the samples were collected at $30 \mathrm{~cm}$-plant height. The highest productions of dry mass of Tanzania grass were obtained with applications of greater N:K and S rates. The highest efficiencies of nitrogen and sulfur conversions were verified with applications of the lowest $\mathrm{N}: \mathrm{K}$ and $\mathrm{S}$ rates, with no significant differences in concentration of these nutrients in the plant tissues as their rate of application increased. Higher $\mathrm{S}$ contensts in leaf tissue and relative recovery of $\mathrm{N}$ and S were observed by applying $450 \mathrm{~kg} / \mathrm{ha} \mathrm{N}: \mathrm{K}$ and $60 \mathrm{~kg} / \mathrm{ha}$ of S.
\end{abstract}

Index terms: nutrient concentration, efficiency of $\mathrm{N}$ and $\mathrm{S}$ conversion, relative $\mathrm{N}$ and $\mathrm{S}$ recuperation, Panicum maximum.

(Recebido para publicação em 14 janeiro de 2004 e aprovado em 30 de novembro de 2004)

\section{INTRODUÇÃO}

Para a exploração intensiva das pastagens é de suma importância a correção da fertilidade dos solos, com o objetivo de alcançar a sustentabilidade na exploração. O nitrogênio é um dos nutrientes mais exigidos pelas plantas forrageiras e sua utilização influencia a produção de massa seca e valor nutritivo da forragem.

De acordo com Werner (1994), a redução da disponibilidade do nitrogênio é uma das principais causas da degradação das pastagens, o que resulta em queda acentuada da capacidade de suporte e do ganho de peso dos animais a cada ano de utilização da pastagem. Relata-se que melhor efeito do nitrogênio tem sido obtido quando as pastagens encontram-se sob disponibilidades razoáveis de enxofre, sendo ambos os nutrientes encontrados em baixas concentrações nos solos do cerrados, pobres em matéria orgânica.

Werner \& Monteiro (1988) relataram que as pastagens com deficiência em nitrogênio apresentaram

\footnotetext{
1. Parte da dissertação de Mestrado da primeira autora.

2. Professora Msc, Departamento de Zootecnia da UCG/UEG - katiazoo@hotmail.com

3. Professor Dr., Departamento de Produção Animal da EV/UFG.

4. Pesquisadores da Embrapa Arroz e Feijão - itamar@cnpaf.embrapa.br

5. Professor Titular Departamento de Solos e Nutrição de Plantas da ESALQ/USP.
} 
baixas respostas ao enxofre. Conseqüentemente, alta disponibilidade de nitrogênio requer aplicação de doses mais altas de enxofre, uma vez que este nutriente é importante no metabolismo do nitrogênio e na síntese de proteína.

A importância do equilíbrio entre as quantidades de nitrogênio e de enxofre no solo e na planta é refletida no crescimento e no estado nutricional do vegetal. A adição de doses mais elevadas de um desses elementos nos sistemas de produção animal pode induzir a menor disponibilidade de um outro elemento para a planta. Com isso, a avaliação da eficiência desses nutrientes constitui um parâmetro importante para o conhecimento do equilíbrio entre as doses de fertilizantes aplicadas no solo com as produções obtidas, constituindo uma ferramenta para melhor entendimento do complexo produtivo com o uso racional das adubações nas atividades agropastoris sustentáveis.

Conduziu-se este trabalho com o objetivo de o efeito da formulação N:K com o uso do S na produção de massa seca (MS) e na eficiência da conversão e na recuperação relativa do nitrogênio e do enxofre no capim-Tanzânia.

\section{MATERIAL E MÉTODOS}

O experimento foi realizado na Fazenda Modelo da Escola de Veterinária da Universidade Federal de Goiás, localizada no Campus II, município de GoiâniaGO com latitude $16^{\circ} 40^{\prime} 00^{\prime \prime} \mathrm{S}$, longitude $49^{\circ} 15^{\prime} 00^{\prime \prime} \mathrm{W}$, altitude de $741 \mathrm{~m}$ e clima tropical quente semi-úmido. Utilizou-se uma área de 12 ha de capim-Tanzânia, dividida em 30 piquetes de 0,4 ha.

A área foi implantada entre janeiro e fevereiro de 2000, quando foram coletadas amostras do solo Latossolo Vermelho Escuro, na profundidade de 0-20 $\mathrm{cm}$. As análises dessas amostras revelaram as seguintes características médias: $\mathrm{pH}$ em água $=5,6 ; \mathrm{Ca}=1,4$ cmolc $/ \mathrm{dm}^{3} ; \mathrm{Mg}=0,4 \mathrm{cmolc} / \mathrm{dm}^{3} ; \mathrm{Al}=0,0 \mathrm{cmolc} / \mathrm{dm}^{3}$; $\mathrm{H}+\mathrm{Al}=2,5 \mathrm{cmolc} / \mathrm{dm}^{3} ; \mathrm{CTC}=4,3 ; \mathrm{P}=1,3 \mathrm{mg} / \mathrm{dm}^{3}$; $\mathrm{K}=30 \mathrm{mg} / \mathrm{dm}^{3} ; \mathrm{Cu}=1,2 \mathrm{mg} / \mathrm{dm}^{3} ; \mathrm{Zn}=0,9 \mathrm{mg} / \mathrm{dm}^{3} ; \mathrm{Fe}$ $=19 \mathrm{mg} / \mathrm{dm}^{3} ; \mathrm{Mn} 40 \mathrm{mg} / \mathrm{dm}^{3} ; \mathrm{MO}=27,0 \mathrm{~g} / \mathrm{dm}^{3}$.

O preparo da área foi realizado utilizando-se o arado de aiveca, e foram aplicados a lanço $\mathrm{P}_{2} \mathrm{O}_{5}$ e FTE BR-16 nas respectivas doses de 80 e $20 \mathrm{~kg} / \mathrm{ha}$, por ocasião da semeadura da pastagem. Utilizou-se uma taxa de semeadura de sementes puras viáveis de 2,5 $\mathrm{kg} / \mathrm{ha}$. Após 40 dias da germinação, foram aplicadas adubações nitrogenadas e potássica de $300 \mathrm{~kg} / \mathrm{ha}$ da formulação 20:00:20, a lanço.
Dez meses depois da implantação do capimTanzânia, o experimento foi instalado num dos piquetes, onde foi realizado um corte de uniformização e em seguida retirado o material vegetal cortado. A área útil utilizada foi de $1080 \mathrm{~m}^{2}$, dividida em 27 parcelas de 40 $\mathrm{m}^{2}$.

Os tratamentos constituíram de 150, 300 e 450 $\mathrm{kg} /$ ha da formulação N:K (20:20), com 20, 40 e 60 $\mathrm{kg} /$ ha de enxofre, empregando-se o gesso agrícola como fonte de S. O delineamento experimental foi de blocos completos ao acaso, em esquema fatorial $3 \times 3$, com três repetições.

O período de avaliação da forrageira foi de seis meses. O primeiro corte foi realizado 40 dias após aplicação dos tratamentos. Foram realizados três cortes no período das águas, com intervalos de 60 dias.

As coletas das amostras foram realizadas com auxílio de um quadrado de ferro com $1 \mathrm{~m}$ x $1 \mathrm{~m}$, e a forragem foi cortada com tesoura de aço à altura de $30 \mathrm{~cm}$ do solo. Após cada corte de avaliação, foi realizado o corte de uniformização de toda a área experimental, a uma altura de $30 \mathrm{~cm}$ do solo, sendo retirado o resíduo.

O material coletado no campo foi acondicionado em saco plástico, identificado e enviado ao laboratório, onde foi pesado e posteriormente retirada uma amostra representativa de cada parcela (aproximadamente 400 g). Em seguida foi colocado em estufa de ventilação forçada de ar, com temperaturas de 58 a $65^{\circ} \mathrm{C}$ por 72 horas, para determinação da massa seca parcial.

Após a secagem, as amostras foram moídas em moinho do tipo Willey, com peneira de $1 \mathrm{~mm}$, armazenadas em sacos de plástico e identificadas. Em seguida, foram realizadas na Embrapa Arroz e Feijão análises químicas foliares para determinação das concentrações de N e S. O N foi determinado por Semimicro-Kjeldahl e o $\mathrm{S}$ por gravimetria do sulfato de bário, conforme a metodologia de Malavolta et al. (1997).

Durante a condução do experimento foram monitorados, diariamente, os dados de: temperatura mínima e máxima, e precipitação pluvial, pela estação meteorológica da Escola de Agronomia da Universidade Federal de Goiás, cujos resultados médios são mostrados na Figura 1.

Os resultados de produção de massa seca e concentração de nitrogênio e enxofre foram analisados de acordo com procedimento GLM do programa estatístico SAS INSTITUTE (1989), comparando-se as médias pelo teste de Tukey. 


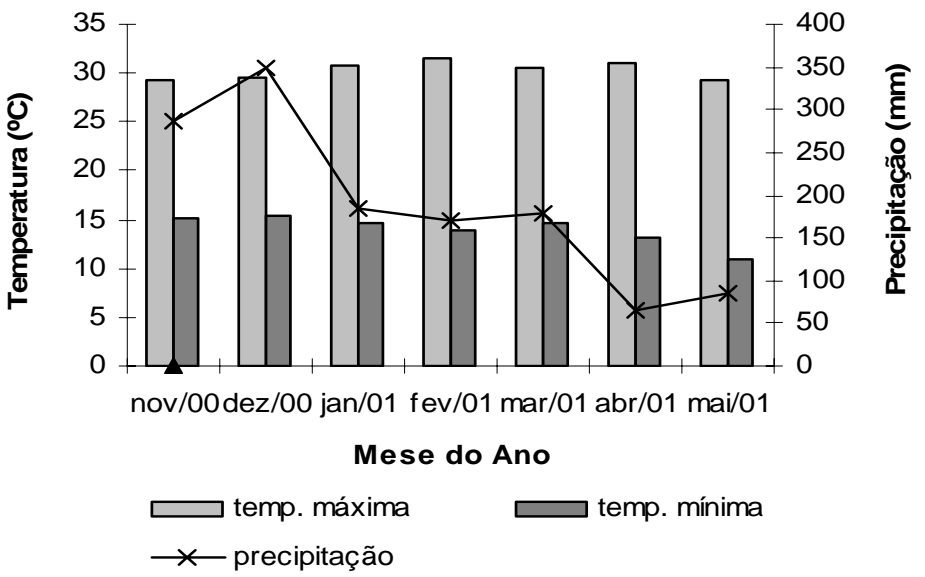

FIGURA 1 - Temperaturas máximas e mínimas $\left({ }^{\circ} \mathrm{C}\right)$ e precipitações pluviais (mm) observadas durante o período de novembro de 2000 a maio de 2001.

A partir dos resultados foi calculada a eficiência de conversão dos nutrientes ( $\mathrm{kg}$ MS / kg de N e S). A quantidade do nutriente contido no tecido da planta foi calculada pela produção de MS (em kg/ha) multiplicada pela concentração média de nitrogênio e enxofre (em kg/ha), dividindo-se por 1000 para expressar em kg/ha.

A recuperação relativa foi calculada a partir do conteúdo de nutriente na dose considerada, em relação à mais baixa aplicada ( $\mathrm{N}: \mathrm{K}=150 \mathrm{~kg} / \mathrm{ha})$.

\section{RESULTADOS E DISCUSSÃO}

O capim-Tanzânia apresentou produções diferenciadas em função dos tratamentos aplicados. Observa-se na Tabela 1 , que houve efeito significativo $(\mathrm{P}<0,05)$ de doses da formulação $\mathrm{N}: \mathrm{K}$ na produção de massa seca. As maiores produções foram verificadas ao aplicar $450 \mathrm{~kg} / \mathrm{ha}$ diferenciando significativamente de 150 e $300 \mathrm{~kg} / \mathrm{ha}$.

Contudo, não foi observado efeito significativo (P > $0,05)$ de doses de $S$, nem significância da interação N:K x S no capim-Tanzânia. Tamassia et al. (1999), trabalhando com capim-Tanzânia, observaram interação significativa entre as doses de $\mathrm{N}$ e $\mathrm{S}$ na produção de MS.

Avaliando a eficiência da conversão do nitrogênio (ECN), observa-se na Tabela 1 que o capimTanzânia apresentou maior ECN quando se aplicaram as menores doses de $\mathrm{N}: \mathrm{K}$, provavelmente, devido ao menor aproveitamento do nitrogênio pelas plantas, resultante de maiores perdas desse nutriente no solo.

Resultados semelhantes foram encontrados por Primavesi et al. (2002), que verificaram redução na eficiência da utilização do nitrogênio, à medida que se aumentaram as doses de $\mathrm{N}$.

Carvalho \& Saraiva (1987) relataram que existe perda de nitrogênio toda vez em que se aplica esse elemento no solo, sendo as mais comuns por meio de volatilização, desnitrificação e, principalmente lixiviação, o que logicamente resulta em baixa taxa da eficiência da utilização do nitrogênio. Possivelmente, essas perdas poderiam ter sido menores se tivessem sido parceladas as adubações após cada corte. De acordo com Werner et al. (2001), as adubações nitrogenadas devem ser parceladas principalmente quando se usam altas doses de nitrogênio, para se evitar principalmente as perdas por volatilização e por lixiviação, para que se obtenha a maior eficiência de utilização desse nutriente pelas plantas forrageiras.

Observa-se na Tabela 1, que o $\mathrm{N}$ contido no tecido da planta (NC) foi maior do que a quantidade de $\mathrm{N}$ aplicada, quando se utilizou 150 e $300 \mathrm{~kg} / \mathrm{ha}$ da formulação $\mathrm{N}: \mathrm{K}$ dentro de todas as doses de $\mathrm{S}$ e 450 $\mathrm{kg} / \mathrm{ha}$ da formulação $\mathrm{N}: \mathrm{K}$ com $60 \mathrm{~kg} / \mathrm{ha}$ de S. Esse resultado é comum porque as plantas podem obter o nitrogênio a partir da atmosfera, daquele mineralizado da matéria orgânica do solo ou do nitrogênio solúvel encontrado na solução do solo que tem a sua disponibilidade aumentada ou diminuída de acordo com a variação da altura do lençol freático.

Segundo Whitehead (1995), a recuperação do nitrogênio pelas gramíneas está entre os limites de 50 a $80 \%$ e com maior freqüência entre 65 a $70 \%$. Com o acréscimo das doses de $\mathrm{N}$, normalmente menos $\mathrm{N}$ é recuperado pelas plantas. 
Produção de massa seca, eficiência e recuperação...

TABELA 1 - Produção de massa seca (MS), eficiência da conversão do nitrogênio (ECN), nitrogênio contido no tecido da planta (NC) e recuperação relativa do nitrogênio (RRN) do capim-Tanzânia, (média de três cortes).

\begin{tabular}{|c|c|c|c|c|c|c|c|c|c|}
\hline \multirow{5}{*}{ Variáveis } & \multicolumn{9}{|c|}{ Tratamentos } \\
\hline & \multicolumn{9}{|c|}{ Formulação N:K 20:20 (kg/ha) } \\
\hline & \multicolumn{3}{|c|}{150} & \multicolumn{3}{|c|}{300} & \multicolumn{3}{|c|}{450} \\
\hline & \multicolumn{9}{|c|}{ Enxofre (kg/ha) } \\
\hline & 20 & 40 & 60 & 20 & 40 & 60 & 20 & 40 & 60 \\
\hline MS (t/ha) & $2,5 \mathrm{Ca}$ & 3,3 Ca & 3,0 Ca & $3,8 \mathrm{Ba}$ & $4,4 \mathrm{Ba}$ & $3,6 \mathrm{Ba}$ & $5,2 \mathrm{Aa}$ & $5,4 \mathrm{Aa}$ & 6,9 Aa \\
\hline N (g/kg) & 14,7 & 14,4 & 16,0 & 16,6 & 16,5 & 15,6 & 16,6 & 15,3 & 18,5 \\
\hline $\mathrm{ECN}(\mathrm{kg} / \mathrm{ha})$ & 83,33 & 110,00 & 100,00 & 63,33 & 73,33 & 60,00 & 57,77 & 60,00 & 76,66 \\
\hline NC (kg/ha) & 36,75 & 47,52 & 48,00 & 63,08 & 72,60 & 56,16 & 86,32 & 82,62 & 127,65 \\
\hline RRN (\%) & - & - & - & 85,71 & 91,30 & 14,40 & 81,60 & 62,50 & 125,58 \\
\hline $\mathrm{CV}(\%)$ & 27,85 & & & & & & & & \\
\hline
\end{tabular}

Médias seguidas de letras iguais indicam que as mesmas não diferem entre si, pelo teste de Tukey (P<0,05). Letras maiúsculas são usadas para comparar doses de nitrogênio e potássio dentro de cada doses de enxofre. Letras minúsculas são usadas para comparar doses de enxofre dentro de cada doses de nitrogênio e potássio.

Quando se aplicou 300 e 450 kg/ha da formulação N:K com 20 e 40 kg/ha de S observa-se na Tabela 1, que ao aumentar as doses de $\mathrm{N}$ diminuiu a RRN. A maior RRN foi observada quando se aplicou $450 \mathrm{~kg} / \mathrm{ha}$ da formulação $\mathrm{N}: \mathrm{K}$ na presença de $60 \mathrm{~kg} / \mathrm{ha}$ de S. Neste tratamento essa recuperação ficou em torno de $125 \%$, resultado muito acima do observado quando se aplicou $300 \mathrm{~kg} /$ ha da formulação N:K com $60 \mathrm{~kg} / \mathrm{ha}$ de $\mathrm{S}$, embora não fosse observada uma relação direta entre doses de $\mathrm{N}: \mathrm{K}$ e S.

Corsi (1994) relatou que a recuperação de nitrogênio pode ser maior de $80 \%$ em gramíneas tropicais, desde que o fertilizante seja adequadamente aplicado.

Não foram observados efeitos significativos ( $\mathrm{P}>$ $0,05)$ das doses de $\mathrm{N}$, ou das doses de $\mathrm{S}$, nem significância para a interação N x S na concentração de S pelo capim-Tanzânia (Tabela 2). Tamassia et al. (1999) observaram que houve aumento na concentração de $\mathrm{S}$ nas folhas em função do enxofre fornecido e esse aumento seguiu um modelo linear, no qual a maior concentração de $\mathrm{N}$ e $\mathrm{S}$ foi obtida nas combinações que receberam as mais altas doses destes elementos na solução.

Pinto et al. (2002), trabalhando com capimTanzânia verificaram concentrações médias de S de 1,4 a $1,5 \mathrm{~g} / \mathrm{kg}$.

A ECS foi maior quando se aplicaram doses mais baixas de $\mathrm{S}$ (Tabela 2). Os mais altos valores foram obtidos quando se aplicou $20 \mathrm{~kg} / \mathrm{ha}$ em relação a 40 e $60 \mathrm{~kg} / \mathrm{ha}$ de S. Outro aspecto importante foi observado com relação às doses de $\mathrm{N}: \mathrm{K}$, sendo os mais altos valores de ECS observados quando se aplicou 450 $\mathrm{kg} / \mathrm{ha}$ de $\mathrm{N}: \mathrm{K}$.

Semelhante ao resultado obtido com a concentração de $\mathrm{S}$, também a maior RRS foi verificada quando se aplicou a maior dose da formulação N:K (450 kg/ha) na presença de $60 \mathrm{~kg} / \mathrm{ha}$ de $S$ (Tabela 2). Embora não fosse observada uma relação direta, devido à variação dos resultados obtidos, pode se dizer que uma adubação necessária para suprir as necessidades fisiológicas da planta e seu potencial de crescimento pode resultar em maiores absorções de $\mathrm{N}$ e $\mathrm{S}$ e maiores produções quando todas as condições do meio são favoráveis ao desenvolvimento vegetal. Monteiro (1986) observou uma recuperação de $S$, entre 7,4 e 4,8 \% quando se aplicou 0 e $45 \mathrm{~kg} / \mathrm{ha}$ de $\mathrm{S}$ respectivamente. Existem poucos estudos sobre a aplicação de $\mathrm{S}$ e sua recuperação em forrageiras, de tal forma que se sugere maior número de trabalhos nesse assunto.

Monteiro \& Carriel (1987) relataram que além da concentração de enxofre no tecido foliar, a relação $\mathrm{N}: \mathrm{S}$ tem sido amplamente utilizada para avaliar o estado nutricional das plantas quanto ao enxofre. A relação N:S encontrada neste trabalho foi de 11:1, com concentrações de S na planta variando entre 1,4 a 1,6 $\mathrm{g} / \mathrm{kg}$. 
TABELA 2 - Concentração de enxofre (S), eficiência da conversão do enxofre (ECS), enxofre contido no tecido da planta (SC) e recuperação relativa do enxofre (RRS) do capim-Tanzânia, (média de três cortes).

\begin{tabular}{|c|c|c|c|c|c|c|c|c|c|}
\hline \multirow{5}{*}{ Variáveis } & \multicolumn{9}{|c|}{ Tratamentos } \\
\hline & \multicolumn{9}{|c|}{ Formulação N:K 20:20 (kg/ha) } \\
\hline & \multicolumn{3}{|c|}{150} & \multicolumn{3}{|c|}{300} & \multicolumn{3}{|c|}{450} \\
\hline & \multicolumn{9}{|c|}{ Enxofre (kg/ha) } \\
\hline & 20 & 40 & 60 & 20 & 40 & 60 & 20 & 40 & 60 \\
\hline $\mathrm{S}(\mathrm{g} / \mathrm{kg})$ & $1,5 \mathrm{Aa}$ & $1,5 \mathrm{Aa}$ & $1,4 \mathrm{Aa}$ & $1,4 \mathrm{Aa}$ & $1,4 \mathrm{Aa}$ & $1,4 \mathrm{Aa}$ & $1,4 \mathrm{Aa}$ & $1,4 \mathrm{Aa}$ & $1,6 \mathrm{Aa}$ \\
\hline ECS (kg/ha) & 125,00 & 82,50 & 50,00 & 190,00 & 110,00 & 60,00 & 260 & 135,00 & 115,00 \\
\hline SC (kg/ha) & 3,75 & 4,95 & 4,20 & 5,32 & 6,16 & 5,04 & 7,28 & 7,56 & 11,04 \\
\hline RRS (\%) & - & 5,85 & 1,20 & - & 6,00 & 0,28 & - & 1,40 & 9,48 \\
\hline CV (\%) & 12,56 & & & & & & & & \\
\hline
\end{tabular}

Médias seguidas de letras iguais indicam que as mesmas não diferem entre si, pelo teste de Tukey $(\mathrm{P}<0,05)$.

Letras maiúsculas são usadas para comparar doses de nitrogênio e potássio dentro de cada dose de enxofre.

Letras minúsculas são usadas para comparar doses de enxofre dentro de cada dose de nitrogênio e potássio.

\section{CONCLUSÕES}

Aplicação de doses maiores de N:K e S resultam em maiores produções de massa seca do capimTanzânia.

Melhores ECN e ECS são observadas quando se aplica menores doses de $\mathrm{N}: \mathrm{K}$ e $\mathrm{S}$, mesmo não havendo diferenças significativas nas concentrações destes nutrientes no tecido da planta sob a ação de doses crescentes dos mesmos. Ao contrário, as maiores RRN e RRS foram observadas quando se aplicou $450 \mathrm{~kg} / \mathrm{ha} \mathrm{da}$ formulação N:K na presença de $60 \mathrm{~kg} / \mathrm{ha}$ de $\mathrm{S}$.

\section{REFERÊNCIAS BIBLIOGRÁFICAS}

CARVALHO, M. M.; SARAIVA, O. F. Resposta do capim-gordura (Melinis minutiflora Beau.) a aplicação de nitrogênio, em regime de cortes. Revista Brasileira de Zootecnia, Viçosa, v. 16, n. 5, p. 442-454, 1987.

CORSI, M. Adubação nitrogenada das pastagens. In: SIMPÓSIO: PASTAGENS - FUNDAMENTOS DA EXPLORAÇÃO RACIONAL, 2., 1994, Piracicaba. Anais... Piracicaba: FEALQ, 1994. p. 121-153.

MALAVOLTA, E.;VITTI, G. C.; OLIVEIRA, S. A. Avaliação do estado nutricional das plantas: princípios e aplicações. 2. ed. Piracicaba: Associação Brasileira da Potassa e do Fosfato, 1997. 319 p.
MONTEIRO, F. A. Sulphur fertilization and nutrient distribution in a Florida spodosol profile under white clover-pensacola Bahiagrass. 1986. $182 \mathrm{f}$. Thesis (PhD) - University of Florida, Florida, 1986.

MONTEIRO, F. A.; CARRIEL, J. M. Aplicação de níveis de enxofre na forma de gesso para o cultivo do capim Colonião em dois solos arenosos do estado de São Paulo. Boletim de Indústria Animal, Nova Odessa, v. 44, n. 2, p. 335-347, 1987.

PINTO, J. C.; BELARMINO, N. C. J.; ROCHA, G. P. Composição mineral da forragem de capim Tanzânia em função da aplicação de superfosfato simples e sulfato de amônio. In: REUNIÃO ANUAL DA SOCIEDADE BRASILEIRA DE ZOOTECNIA, 39., 2002, Recife. Anais... Recife: Sociedade Brasileira de Zootecnia, 2002. CD-ROM.

PRIMAVESI, O.; CORRÊA, L. A.; PRIMAVESI, A. C.; SILVA, A. G.; CANTARELlA, H. Eficiência nutricional de duas fontes de nitrogênio na produção de matéria seca de capim-Marandu. In: REUNIÃO ANUAL DA SOCIEDADE BRASILEIRA DE ZOOTECNIA, 39., 2002, Recife. Anais... Recife: Sociedade Brasileira de Zootecnia, 2002. CD-ROM.

SAS INSTITUTE. Statistical analysis system: language and procedures. Version 6. Cary, 1989. 
TAMASSIA, L. F. M.; MONTEIRO, F. A.; MANARIN, C. A.; GUIMARÃES, G. P. F. P. B.; PREMAZZI, L. M. Interação entre doses de nitrogênio e de enxofre para o estabelecimento e perfilhamento do capim Tanzânia. In: REUNIÃO ANUAL DA SOCIEDADE BRASILEIRA DE ZOOTECNIA, 36., Porto Alegre, 1999. Anais... Porto Alegre: Sociedade Brasileira de Zootecnia, 1999. CD ROM.

WERNER, J. C. Adubação de pastagens de Brachiaria spp. In: SIMPÓSIO SOBRE MANEJO DE PASTAGEM, 11., 1994, Piracicaba. Anais... Piracicaba: FEALQ, 1994. p. 209-266.
WERNER, J. C.; MONTEIRO, F. A. Respostas das pastagens a aplicação de enxofre. In: SIMPÓSIO SOBRE ENXOFRE E MICRONUTRIENTES NA AGRICULTURA BRASILEIRA, 1988, Londrina. Anais... Londrina: [s.n.], 1988. p. 87-99.

WERNER, J. C.; COLOZZA, M. T.; MONTEIRO, F. A. Adubação de pastagens. In: SIMPÓSIO SOBRE MANEJO DE PASTAGENS, 18., 2001, Piracicaba. Anais... Piracicaba: FEALQ, 2001. p. 129-156.

WHITEHEAD, D. C. Volatilization of ammonia. In: . Grassland nitrogen. Wallingford: CAB International, 1995. p. 152-179. 